\title{
Evaluation of the Effect of Aspirin on Platelet Aggregation: Methodological Recommendations for Aspirin-Drug Interaction Studies
}

\author{
Annelieke C. Kruithof ${ }^{1}$, Matthijs Moerland', Eleftheria A. Anastasopoulou', \\ Pieter-Jan de Kam², Marieke L. de Kam³ ${ }^{3}$ Jacobus Burggraaf' \\ ${ }^{1}$ Department of Vascular Medicine, Centre for Human Drug Research, Leiden, The Netherlands \\ ${ }^{2}$ Department of Clinical Pharmacology, Merck Sharp \& Dohme Corp., Rahway, USA \\ ${ }^{3}$ Department of Pharmacometrics, Centre for Human Drug Research, Leiden, The Netherlands \\ Email: akruithof@chdr.nl
}

Received 24 December 2014; accepted 10 January 2015; published 19 January 2015

Copyright (C) 2015 by authors and Scientific Research Publishing Inc.

This work is licensed under the Creative Commons Attribution International License (CC BY). http://creativecommons.org/licenses/by/4.0/

(c) (i) Open Access

\begin{abstract}
Given the broad application of aspirin as antiplatelet drug, availability of standardized methodology to assess potential interaction with any co-medication on platelet aggregation is desired. We characterized the effect of aspirin (ASA) therapy on collagen-induced platelet aggregation in whole blood to define such methodology. Collagen-induced platelet whole blood aggregation was assessed in 6 healthy male volunteers on 2 occasions (Day 1, Day 7) using the Chronolog aggregometer. From Day 2 up to Day 7, subjects received a daily oral dose of $75 \mathrm{mg}$ ASA. The relationship between collagen dose and platelet aggregation response was assessed. On Day 1, maximal aggregation was observed at $1 \mu \mathrm{g} / \mathrm{mL}$ collagen $(15.3 \pm 4.6 \Omega)$ and higher. Reproducible results were obtained without any indication of intra-subject fluctuations. ASA treatment decreased maximal aggregation by $80 \%$ and $38 \%$ at 0.5 and $2.0 \mu \mathrm{g} / \mathrm{mL}$ collagen, respectively. Power calculations were performed based on the observed intra-subject variability and demonstrated minimal sample sizes of 9 - 11 subjects for future cross-over ASA-drug interaction studies exploring effects on platelet aggregation, which demonstrates that the proposed collagen-induced ex vivo whole blood platelet aggregation is a feasible methodology to evaluate ASA-drug interactions in healthy volunteers.
\end{abstract}

\section{Keywords}

Platelet Aggregation, Impedance Aggregometry, Whole Blood, Collagen, Aspirin Interaction 


\section{Introduction}

Aspirin (acetylsalicylic acid; ASA) is the most widely used platelet function inhibitor [1]. Low ASA doses of 75 - 325 $\mathrm{mg} /$ day are prescribed for prevention of thrombotic cardiovascular disease as recommended by the American College of Chest Physicians (ACCP) [2]. The number of patients on long-term ASA treatment receiving a wide variety of concomitant drugs, such as statins and angiotensin-converting-enzyme (ACE) inhibitors, is relatively high [3]. Therefore, availability of standardized methodology evaluating the potential interaction between ASA and any co-medication on platelet function is desired. Ideally, such methodology should represent human physiology, be reproducible, have minimal intra-subject variation, and provide a sufficient window for evaluations of changes in platelet function.

A frequently used method for quantification of the effect of antiplatelet drugs is ex vivo platelet aggregation in response to an agonist, so called platelet aggregometry. The preferred matrix for this method is whole blood rather than platelet rich plasma (PRP) since whole blood has no need of sample centrifugation which may introduce artifacts and it more closely resembles the real-life physiologic conditions, due to the presence of red blood cells and other cell types [4]-[6]. Using whole blood aggregometry, different platelet agonists can be used to assess the inhibitory effect of ASA, either alone or in combination with other anti-aggregant agents, such as arachidonic acid (AA), collagen, adenosine phosphate (ADP) and adrenaline. Generally, 1 relatively high agonist concentration is selected to activate platelets [7]-[12]. This approach is particularly useful to discriminate between ASA responders and non-responders. However, for studying potential ASA-drug interactions this experimental setup is less feasible since induction of a maximal platelet aggregation level does not allow demonstration of drug-induced to increase or to subtle decrease in the aggregatory response.

Therefore, we aimed to develop a platelet aggregometry methodology feasible for evaluation of potential interaction between ASA and any drug-of-choice, which should facilitate rational design of ASA-drug interaction studies. For this purpose, we characterized the modulation of whole blood platelet aggregation by ASA therapy. We selected collagen as aggregation inducer since it played a key role in the initial stage of the haemostatic cascade in vivo, i.e. the interaction of platelets with the extracellular matrix (ECM) of an injured vascular wall. Collagen is the most abundant thrombogenic component present in ECM and its interaction with platelets triggers platelet activation and subsequently platelet adhesion and aggregation [13]. Collagen-induced whole blood platelet aggregometry is a well-known method to assess ASA responsiveness, to discriminate between ASA responders and non-responders and to determine efficacy of dual ASA therapy [14] [15], however, it has not been evaluated for assessment of ASA-drug interactions. Therefore, we assessed the relationship between collagen concentration and platelet aggregation response in a relatively small group of healthy male volunteers, since this was a common population for drug-drug interaction studies. Assay reproducibility and intra-subject variability in the aggregatory response were explored to assess method robustness. The effect of ASA treatment on collagen-induced ex vivo whole blood platelet aggregation (amplitude, lag time and aggregation rate) was evaluated, with the aim to define the methodological conditions inducing a level of aggregation offering sufficient window for demonstration of the presence or absence of additional effects of any drug-of-choice in an ASA interaction study. Based on the observed variability, power calculations were performed to estimate desired sample sizes for future ASA-drug interaction studies.

\section{Methods and Materials}

The study was approved by the Medical Ethics Committee of Leiden University Medical Center, The Netherlands, and conducted in compliance with Dutch law on experiments in humans. All subjects gave written informed consent.

\subsection{Study Population}

Six healthy male volunteers aged 18 - 55 years participated in the study, with a Body Mass Index (BMI) between 18 and $32 \mathrm{~kg} / \mathrm{m}^{2}$ and a normal activated Partial Thromboplastin Time (aPTT), Prothrombin Time (PT) and bleeding time at screening, and no clinically significant findings in (family) medical history (e.g. hematological disorders), physical examination and laboratory tests. The use of concomitant medication was prohibited. 


\subsection{Study Design}

This open-label study was conducted in a study period consisting of 7 days. On Day 1 and Day 7, subjects stayed at the clinical research unit for assessment of platelet aggregation. From the morning of Day 2 until Day 7 (6 consecutive days), subjects visited the clinical research unit daily for supervised oral administration of $75 \mathrm{mg}$ ASA (Actavis). On Day 1 (before start of ASA treatment) and Day 7 (during ASA treatment), blood samples were taken at -5 min, 45 min and 1, 1.5, 3.5 and 6.5 hours. $\mathrm{T}=0$ on Day 1 and Day 7 were comparable actual clock times, but only on $t=0$ at Day 7 ASA was administered. Blood was collected in $5.0 \mathrm{~mL}$ tubes containing 1/10 citrate solution (Sarstedt, Nümbrecht, Germany) using a free-flow 18 gauge intravenous catheter without venous occlusion (Bection Dickinson, Franklin Lanes, USA) after discarding the first $0.5 \mathrm{~mL}$ blood.

\subsection{Platelet Aggregation}

Platelet aggregation was assessed using the Chronolog 590-X whole blood impedance aggregometer (ChronoLog, Corp., PA, USA). Platelet aggregation was induced by rising collagen concentrations $(0.5,0.75,1$ and 2 $\mu \mathrm{g} / \mathrm{mL}$; type 1 fibrils, Chrono-Par reagents, Chrono-Log Corp., stock solution $1 \mathrm{mg} / \mathrm{mL}$, diluted in isotonic glucose solution at $\mathrm{pH} 2.7$ - 2.9). Measurements of platelet aggregation were performed in duplicate for each collagen concentration. Addition of collagen to whole blood sample corresponded with the start of recording an impedance curve which was allowed to run for 15 minutes or until a constant amplitude have been reached. Aggregometry results were expressed as maximum amplitude (resembling maximal aggregation) and recorded in Ohm $(\Omega)$. The maximal slope of the aggregation curve was automatically calculated from the tangents at the steepest slope of the aggregation curve and expressed in $\Omega / \mathrm{min}$. The time period required for the onset of aggregation after collagen addition (lag time) was automatically measured and expressed in seconds.

\subsection{Data Analysis}

Assay reproducibility was assessed by calculation of the coefficient of variation of duplicate aggregation measurements. All subsequent analyses were based on the first data point of each duplicate measurement set, which should fall within the predefined acceptable assay limit of $15 \%$. Intra-individual variability in platelet aggregation (amplitude) was assessed on Day 1 (no ASA) and on Day 7 (on ASA-treatment). For assessment of ASA effects on platelet aggregation, the average maximal aggregation amplitude was calculated for each collagen concentration based on the first time point on Day 1 (pre-ASA) and the first time point of Day 7 (post-ASA, before final ASA administration). All data are presented as mean \pm standard deviation unless indicated otherwise. A power calculation was performed to estimate the desired sample sizes for future ASA-drug interaction studies with a cross-over design, based on the observed intra-individual variability in aggregation amplitude on Day 7. A log-normal data distribution was assumed, and the calculation was performed at $90 \%$ nominal power to demonstrate a treatment effect of $50 \%$ and two-sided significance level of $5 \%$.

\section{Results}

\subsection{Induction of Whole Blood Platelet Aggregation by Collagen}

The relationship between collagen concentration and platelet aggregation response (defined by aggregation amplitude) was assessed in fresh whole blood samples from 6 healthy volunteers (Figure 1). A maximal aggregation response of approximately $15-16 \Omega$ was reached at 1.0 and $2.0 \mu \mathrm{g} / \mathrm{mL}$ collagen (Figure 1 ). Assay reproducibility was adequate for all collagen concentrations tested, with a coefficient of variation of duplicate measurements within the predefined acceptable assay limit of $15 \%$ (data not shown). Over the 6.5 hours observation period on Day 1, intra-subject variability was dependent on the applied collagen concentration, with the lower observed variability at higher collagen concentrations. The intra-subject CV was $22.7 \%$ at $0.5 \mu \mathrm{g} / \mathrm{mL}$ collagen and $9.9 \%$ at $2.0 \mu \mathrm{g} / \mathrm{mL}$ collagen. The response in aggregation rate mirrored the response in aggregation amplitude while increasing collagen concentrations decreased the aggregation lag time (data not shown).

\subsection{Inhibition of Collagen-Induced Platelet Aggregation by ASA}

Daily administration of $75 \mathrm{mg}$ ASA for 5 consecutive days strongly reduced maximal platelet aggregation, as expressed in amplitude, for collagen concentrations between 0.5 and $2.0 \mu \mathrm{g} / \mathrm{mL}$. At low collagen concentrations, 
platelet aggregation was almost completely inhibited by ASA treatment (Figure 1, reduction from $9.0 \pm 5.3 \Omega$ to $1.8 \pm 0.4 \Omega$ at $0.5 \mu \mathrm{g} / \mathrm{mL}$ collagen). At higher collagen concentrations, the ASA-induced reduction in platelet aggregation was less pronounced (Figure 1 , from $15.3 \pm 4.6 \Omega$ to $4.2 \pm 0.8 \Omega$ at $1.0 \mu \mathrm{g} / \mathrm{mL}$ collagen, and from $15.5 \pm 2.4 \Omega$ to $9.7 \pm 4.6 \Omega$ at $2.0 \mu \mathrm{g} / \mathrm{mL}$ collagen).

The effect of ASA therapy on collagen-induced platelet aggregation was also assessed by aggregation rate and lag time. Aggregation rate showed an ASA effect pattern that was comparable with the pattern observed for aggregation amplitude and lag time followed a similar, but reverse pattern (data not shown).

Any additional effect of the last ASA administration on Day 7 on platelet aggregation was not observed: ASA administration for 5 consecutive days had already resulted in a maximal inhibition of aggregation for all parameters tested (data not shown). The observed intra-subject variability for platelet aggregation amplitude on ASA treatment (assessed over the course of Day 7) was 36.7\% and 32.5\% for 1 and $2 \mu \mathrm{g} / \mathrm{mL}$ collagen, respectively (Table 1). Based on these variabilities, power calculations were performed demonstrating that group sizes of 11 and 9 subjects (for 1 and $2 \mu \mathrm{g} / \mathrm{mL}$ collagen, respectively) would be required to demonstrate a treatment effect of $50 \%$ in future ASA-drug interaction studies with a cross-over design (Table 1).

\section{Discussion}

Routine low dose ASA therapy is frequently applied as cardiovascular disease prophylaxis [14]. This therapy is mostly long-term and concomitant medication use is almost inevitable [3]. Hence a methodology to evaluate potential interactions affecting platelet function between ASA and co-medication is desired. We developed and characterized a platelet aggregometry methodology for assessment of potential ASA-drug interactions affecting platelet aggregation. We chose an experimental setup that optimally represents human physiology (whole blood as matrix, collagen as inducer), and demonstrated method robustness (in terms of assay reproducibility and intrasubject variability). As such, the desired criteria for methodology evaluating potential interactions between ASA

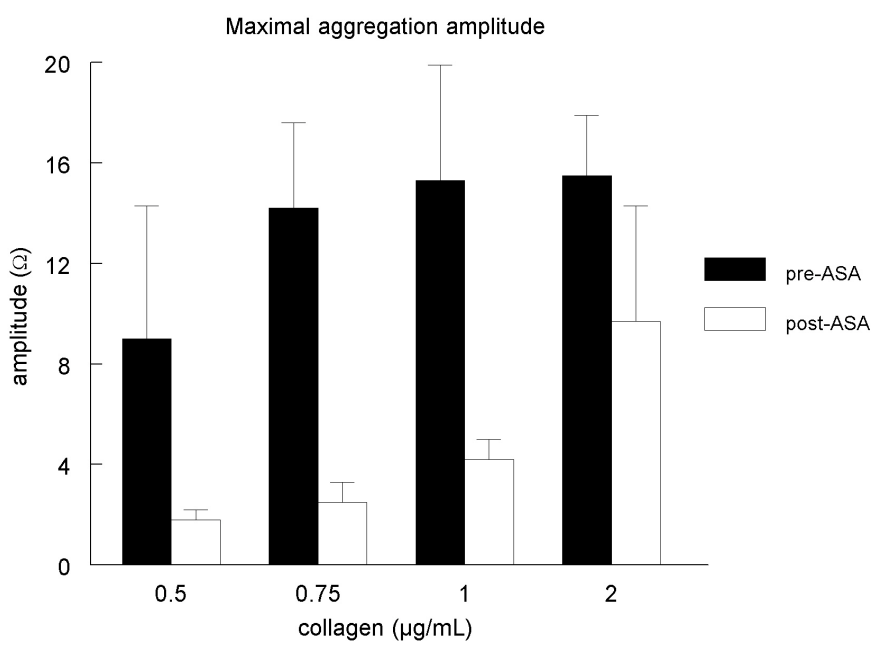

Figure 1. Mean maximal aggregation expressed as amplitude of impedance $(\Omega)$ before ASA administration (pre-ASA) and after $75 \mathrm{mg}$ ASA administration once daily for 5 consecutive days (post-ASA) in response to varying collagen concentrations. Error bars represent standard deviation, $\mathrm{n}=6$.

Table 1. Power calculation for ASA-drug interaction studies with a cross-over design assessing amplitude of platelet aggregation at 1 or $2 \mu \mathrm{g} / \mathrm{mL}$ collagen, based on the intra-subject variability post-ASA $(n=6)$ assuming a log-normal distribution, at $90 \%$ nominal power to demonstrate a treatment effect of $50 \%$ and two-sided significance level of $5 \%$.

\begin{tabular}{cccc}
\hline $\begin{array}{c}\text { Collagen concentration } \\
(\mu \mathrm{g} / \mathrm{mL})\end{array}$ & $\begin{array}{c}\text { Intra-subject CV } \\
\text { post-ASA }(\%)\end{array}$ & Actual power $(\%)$ & Sample size \\
\hline 1.0 & 36.7 & 91.8 & 11 \\
2.0 & 32.5 & 92.5 & 9 \\
\hline
\end{tabular}


and any other drug in a clinical study were met.

A range of collagen concentrations was selected to induce platelet aggregation in fresh whole blood samples including a concentration of $1.0 \mu \mathrm{g} / \mathrm{mL}$ which is often used in the clinical assessment of ASA effects [15] [16]. A clear relationship between collagen concentration and aggregation amplitude response was observed, with an aggregation level of $9 \Omega$ at $0.5 \mu \mathrm{g} / \mathrm{mL}$ and a maximal aggregation of $15-16 \Omega$ at 1.0 and $2.0 \mu \mathrm{g} / \mathrm{mL}$. Inter-individual differences in responsiveness to collagen were observed, with higher variability at lower collagen concentrations. This can be attributed to the fact that 2 subjects showed a low aggregation at $0.5 \mu \mathrm{g} / \mathrm{mL}$ collagen, whereas the other 4 subjects had already (sub-)maximal aggregation at this collagen concentration. These results suggest the existence of an individual collagen threshold concentration that triggers platelet aggregation to exceed a certain basal level (approximately 1 - $5 \Omega$ ) via an all-or-nothing response; when the collagen stimulus is strong enough, a platelet aggregation response is evoked and augmented through the strong self-amplifying loops of the haemostatic cascade. This is consistent with Kawasaki et al. who described comparable differences in platelet sensitivity to collagen in PRP [17], and our earlier findings [6].

We demonstrated that platelet aggregation was effectively inhibited after 6 days of daily $75 \mathrm{mg}$ ASA treatment, which is in line with other studies [7] [18]. The degree of inhibition by ASA treatment was determined by the collagen concentration and ranged from $80 \%$ to $38 \%$ (for 0.5 and $2.0 \mu \mathrm{g} / \mathrm{mL}$ collagen respectively).We also assessed the effect of ASA treatment on platelet aggregation by slope (aggregation rate) and lag time (aggregation onset), but found that for assessment of ASA effects all three readout parameters were generally interchangeable. This is consistent with the mode of action of ASA. Since amplitude is the most frequently used readout parameter of platelet aggregation, we considered this parameter to be the preferred parameter for the future ASA-drug interaction studies. The desired level of platelet aggregation, assessed by amplitude, for ASA-drug interaction studies should allow demonstration of both drug-induced reductions and elevations on an ASA background. Since lower collagen concentrations of 0.5 and $0.75 \mu \mathrm{g} / \mathrm{mL}$ result in minimal platelet aggregation levels in ASA-treated volunteers, these concentrations are not suitable from a methodological perspective. In contrast, higher collagen concentrations ( 1 to $2 \mu \mathrm{g} / \mathrm{mL}$ ) do offer a sufficient window for demonstration of potential drug-induced changes in platelet aggregation. The observed intra-subject variability in aggregation amplitude at 1 and $2 \mu \mathrm{g} / \mathrm{mL}$ collagen on Day 7 was used to assess the required sample size for future ASA-drug interaction studies with a cross-over design. A sample size of 11 and 9 subjects would provide sufficient power to detect a $50 \%$ treatment effect at 1 and $2 \mu \mathrm{g} / \mathrm{mL}$ collagen, respectively. A recent ASA-sugammadex interaction study that applied the described methodology that we describe here, confirms the reported intra-subject variability and estimated sample size [19]. The observed intra-subject CV in this study was $20 \%-23 \%$, with an actual sample size of 23 subjects, at a collagen concentration of $1.5 \mu \mathrm{g} / \mathrm{mL}$. This variability was sufficiently low to confirm the absence of an ASA-sugammadex interaction, defined as a 33\% further inhibition in platelet aggregation. This demonstrates that the findings in our relatively small sample size of 6 subjects can be translated to larger sample sizes, and that our collagen-induced whole blood aggregation methodology is feasible for evaluation of ASAdrug interactions.

\section{Conclusion}

Overall, our experiments support the feasibility of collagen-induced whole blood aggregation to evaluate ASAdrug interactions in healthy male volunteers. The methodology that we developed optimally represents human physiology, and demonstrated method robustness in terms of assay reproducibility and intra-subject variability. Based on our experiments and subsequent power calculations, a collagen concentration of $1-2 \mu \mathrm{g} / \mathrm{mL}$ and a minimal group size of 9 - 11 subjects should be selected for future cross-over ASA-drug interaction studies assessing platelet aggregation effects of a drug-of-choice. This setup will result in a platelet aggregation level (expressed in aggregation amplitude) that offers sufficient window for demonstration of drug-induced changes.

\section{Acknowledgements}

This research was financially supported by Merck Sharp \& Dohme Corp., Whitehouse Station, NJ, USA.

\section{References}

[1] De Meyer, S.F., Vanhoorelbeke, K., Broos, K., Salles, I.I. and Deckmyn, H. (2008) Antiplatelet Drugs. British Journal 
of Haematology, 142, 515-528. http://dx.doi.org/10.1111/j.1365-2141.2008.07233.x

[2] Guyatt, G.H., Akl, E.A., Crowther, M., Gutterman, D.D. and Schünemann, H.J., The American College of Chest Physicians Antithrombotic Therapy and Prevention of Thrombosis Panel (2012) Executive Summary: Antithrombotic Therapy and Prevention of Thrombosis, 9th ed: American College of Chest Physicians Evidence-Based Clinical Practice Guidelines. Chest, 141, 7S-47S. http://europepmc.org/articles/pmc3278060

[3] Feher, G., Koltai, K., Papp, E., Alkonyi, B., Solyom, A., Kenyeres, P., et al. (2006) Aspirin Resistance-Possible Roles of Cardiovascular Risk Factors, Previous Disease History, Concomitant Medications and Haemorrheological Variables. Drugs Aging, 23, 559-567. http://dx.doi.org/10.2165/00002512-200623070-00002

[4] Cardinal, D.C. and Flower, R.J. (1980) The Electronic Aggregometer: A Novel Device for Assessing Platelet Behavior in Blood. Journal of Pharmacological Methods, 3, 135-158. http://dx.doi.org/10.1016/0160-5402(80)90024-8

[5] Dyszkiewicz-Korpanty, A.M., Frenkel, E.P. and Sarode, R. (2005) Approach to the Assessment of Platelet Function: Comparison between Optical-Based Platelet-Rich Plasma and Impedance-Based Whole Blood Platelet Aggregation Methods. Clinical and Applied Thrombosis/Hemostasis, 11, 25-35. http://dx.doi.org/10.1177/107602960501100103

[6] Moerland, M., Kemme, M.J., van der Linden, M. and Burggraaf, J. (2010) Measurement of Collagen- and SerotoninInduced Platelet Aggregation in Whole Blood. Expert Review of Clinical Pharmacology, 3, 177-182. http://dx.doi.org/10.1586/ecp.10.2

[7] de la Cruz, J.P., Camara, S., Bellido, I., Carrasco, T. and Sanchez de la Cuesta, F. (1987) Platelet Aggregation in Human Whole Blood after Chronic Administration of Aspirin. Thrombosis Research, 46, 133-140. http://dx.doi.org/10.1016/0049-3848(87)90213-1

[8] Ivandic, B.T., Giannitsis, E., Schlick, P., Staritz, P., Katus, H.A. and Hohlfeld, T. (2007) Determination of Aspirin Responsiveness by Use of Whole Blood Platelet Aggregometry. Clinical Chemistry, 53, 614-619. http://dx.doi.org/10.1373/clinchem.2006.081059

[9] McGlasson, D.L. and Fritsma, G.A. (2008) Comparison of Four Laboratory Methods to Assess Aspirin Sensitivity. Blood Coagulation \& Fibrinolysis, 19, 120-123. http://dx.doi.org/10.1097/MBC.0b013e3282f40dc2

[10] Meves, S.H., Neubauer, H., Overbeck, U. and Endres, H.G. (2011) Is There an Ideal Way to Initiate Antiplatelet Therapy with Aspirin? A Crossover Study on Healthy Volunteers Evaluating Different Dosing Schemes with Whole Blood Aggregometry. BMC Research Notes, 4, 1-8. http://www.biomedcentral.com/1756-0500/4/106/

[11] Neubauer, H., Kaiser, A.F.C., Endres, H.G., Kruger, J.C., Engelhardt, A., Lask, S., et al. (2011) Tailored Antiplatelet Therapy Can Overcome Clopidogrel and Aspirin Resistance-The BOchum CLopidogrel and Aspirin Plan (BOCLAPlan) to Improve Antiplatelet Therapy. BMC Medicine, 9, 1-12. http://www.biomedcentral.com/1741-7015/9/3/

[12] Spectre, G., Arnetz, L., Ostenson, C.G., Brismar, K., Li, N. and Hjemdahl, P. (2011) Twice Daily Dosing of Aspirin Improves Platelet Inhibition in Whole Blood in Patients with Type 2 Diabetes Mellitus and Micro- or Macrovascular Complications. Thrombosis and Haemostasis, 106, 491-499. http://dx.doi.org/10.1160/TH11-04-0216

[13] Nieswandt, B. and Watson, S.P. (2003) Platelet-Collagen Interaction: Is GPVI the Central Receptor? Blood, 102, 449461. http://dx.doi.org/10.1182/blood-2002-12-3882

[14] Campbell, C.L., Smyth, S., Montalescot, G. and Steinhubl, S.R. (2007) Aspirin Dose for the Prevention of Cardiovascular Disease-A Systematic Review. JAMA, 297, 2018-2024. http://dx.doi.org/10.1001/jama.297.18.2018

[15] McGlasson, D. and Fritsma, G. (2009) Whole Blood Platelet Aggregometry and Platelet Function Testing. Seminars in Thrombosis and Hemostasis, 35, 168-180. http://dx.doi.org/10.1055/s-0029-1220325

[16] Breddin, H.K. (2005) Can Platelet Aggregometry Be Standardized? Platelets, 16, 151-158. http://dx.doi.org/10.1080/09537100400020161

[17] Kawasaki, T., Ozeki, Y., Igawa, T. and Kambayashi, J. (2000) Increased Platelet Sensitivity to Collagen in Individuals Resistant to Low-Dose Aspirin. Stroke, 31, 591-595. http://dx.doi.org/10.1161/01.STR.31.3.591

[18] Kariyazono, H., Nakamura, K., Arima, J., Ayukawa, O., Onimaru, S., Masuda, H., et al. (2004) Evaluation of Anti-Platelet Aggregatory Effects of Aspirin, Cilostazol and Ramatroban on Platelet-Rich Plasma and Whole Blood. Blood Coagulation \& Fibrinolysis, 15, 157-167. http://dx.doi.org/10.1097/00001721-200403000-00007

[19] de Kam, P.J., El Galta, R., Kruithof, A.C., Fennema, H., van Lierop, M.J., Mihara, K., et al. (2013) No Clinically Relevant Interaction between Sugammadex and Aspirin on Platelet Aggregation and Coagulation Parameters. International Journal of Clinical Pharmacology and Therapeutics, 51, 976-985. http://dx.doi.org/10.5414/CP201970 
Scientific Research Publishing (SCIRP) is one of the largest Open Access journal publishers. It is currently publishing more than 200 open access, online, peer-reviewed journals covering a wide range of academic disciplines. SCIRP serves the worldwide academic communities and contributes to the progress and application of science with its publication.

Other selected journals from SCIRP are listed as below. Submit your manuscript to us via either submit@scirp.org or Online Submission Portal.
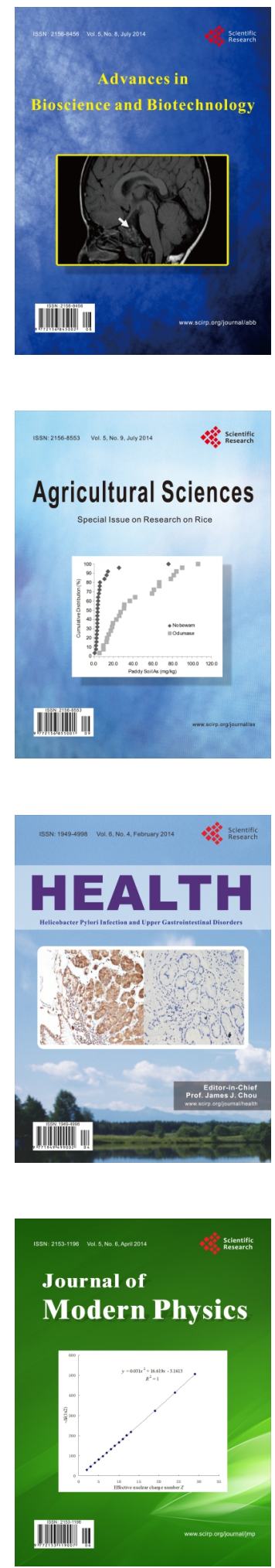
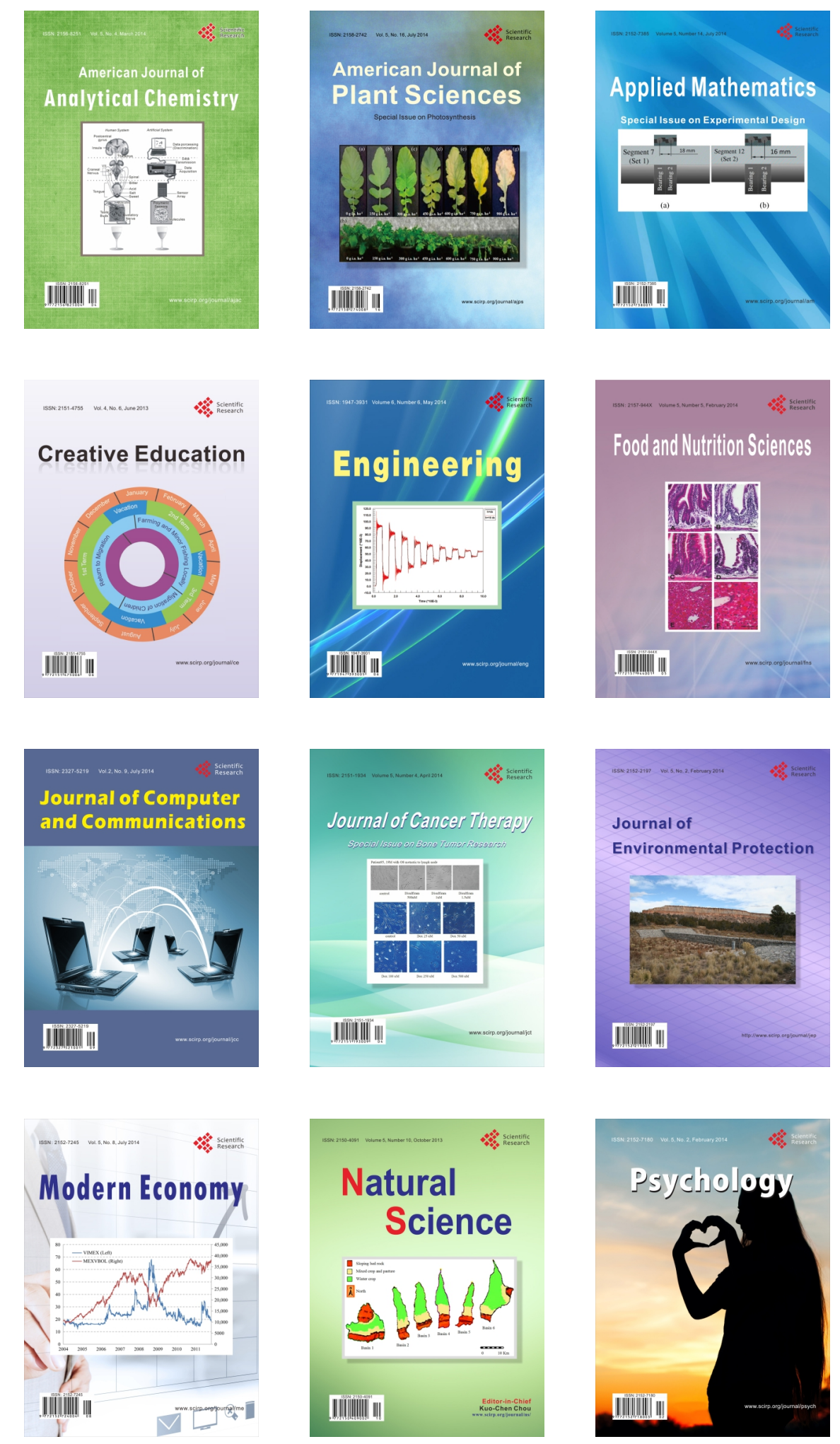